\title{
A True Tale of a False Tail: A rare case of Vestigial Tail with Spina Bifida
}

\author{
Nikhil Naithottu G, Venkatesh M Annigeri,* Akshay Kalavant B, Anil B Halgeri
}

Department of Pediatric Surgery, SDM College of Medical Sciences \& Hospital, India

How to cite: Naithottu G N, Annigeri VM, Kalavant B A, Halgeri AB. A true tale of a false tail: A rare case of vestigial tail with spina bifida. J Neonatal Surg. 2017;6:81.

This is an open-access article distributed under the terms of the Creative Commons Attribution License, which permits unrestricted use, distribution, and reproduction in any medium, provided the original work is properly cited.

\begin{abstract}
A human tail is a rare congenital anomaly with a prominent lesion in the lumbosacrococcygeal region. True tail does not have any underlying spinal abnormalities, whereas pseudo tails are associated with spinal abnormalities. It requires radiological investigation to differentiate between two and treat accordingly. Long term follow-up is required in case of pseudo tail to assess neurological development. Only few cases have been reported in English literature of vestigial tail with spina bifida. Here we report a case of vestigial tail with spina bifida in a newborn born to a mother with gestational diabetes mellitus.
\end{abstract}

Key words: Vestigial tail; Spina bifida; Caudal appendage; Pseudo tail

\section{CASE REPORT}

A baby girl (4kg) born to a married couple with second degree of consanguinity, presented at day one of life for a tail like extension at sacral region with swelling at the end of the lesion (Fig.1A). Baby had cried immediately after birth and APGAR was 7/10 and 8/10 at one minute and five minute respectively. Mother's obstetric score was Gravida 4 Parity 2 Living 1 Abortion 2 and she was diagnosed with GDM. Mother had irregular antenatal visits and did not undergo antenatal ultrasonography. She was taking folic acid supplementation regularly during her pregnancy. Clinical examination of baby revealed 12 centimeter long tail like extension at sacral region with a ovoid shaped swelling at the end of the tail. The ovoid swelling was $4 \times 4 \times 5$ centimeter, soft in consistency and skin over the swelling was normal (Fig.1A). The primitive reflexes of the baby were normal. Systemic examination revealed no abnormality. At day one of age baby underwent neurosonology which was normal. MRI of lumbosacral spine (Fig 2A,B) revealed spina bifida at S3, S4 and S5 levels, low lying tethered cord with conus at L5-S1 level, well defined soft tissue signal intensity lesion appearing mixed intense on $\mathrm{T} 1 \mathrm{~W}$ and predominantly hyperintense T2W. Majority of lesion is seen external to skin surface with small portion attached to skin and subcutaneous tissue at S3-S4 level. Few fibrous bands were seen connecting the lesion with spinal canal.

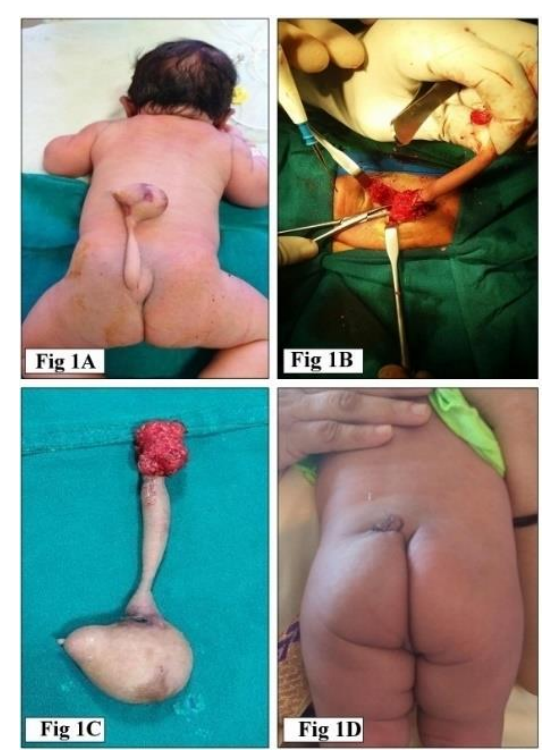

Figure 1: A) Preoperative - Showing baby with a $12 \mathrm{~cm}$ long tail. B) Intra operative - Elliptical incision taken around the vestigial tail.

C) Excised specimen. D) Postoperative - healthy wound.

Hematological investigations were normal. On day three of life the baby was taken up for surgery. Elliptical incision was placed around the base of the tail 
(Fig.1B), tract was dissected, defect noted underneath was closed and the tail was excised (Fig.1C). Wound closed in layers. Histopathology of the globular mass specimen showed stroma consisting of lobules of adipocytes with pilosebaceous unit, congested blood vessels, nerve bundles, bone marrow with trabecular bones, glial tissue, skeletal muscle fibers. Specimen of stalk showed skeletal muscle fibres with adipose tissue and congested blood vessels. Postoperative course was uneventful, baby is doing well after 1 month of follow-up with good cosmetic results (Fig.1D) and without any neurological deficit.

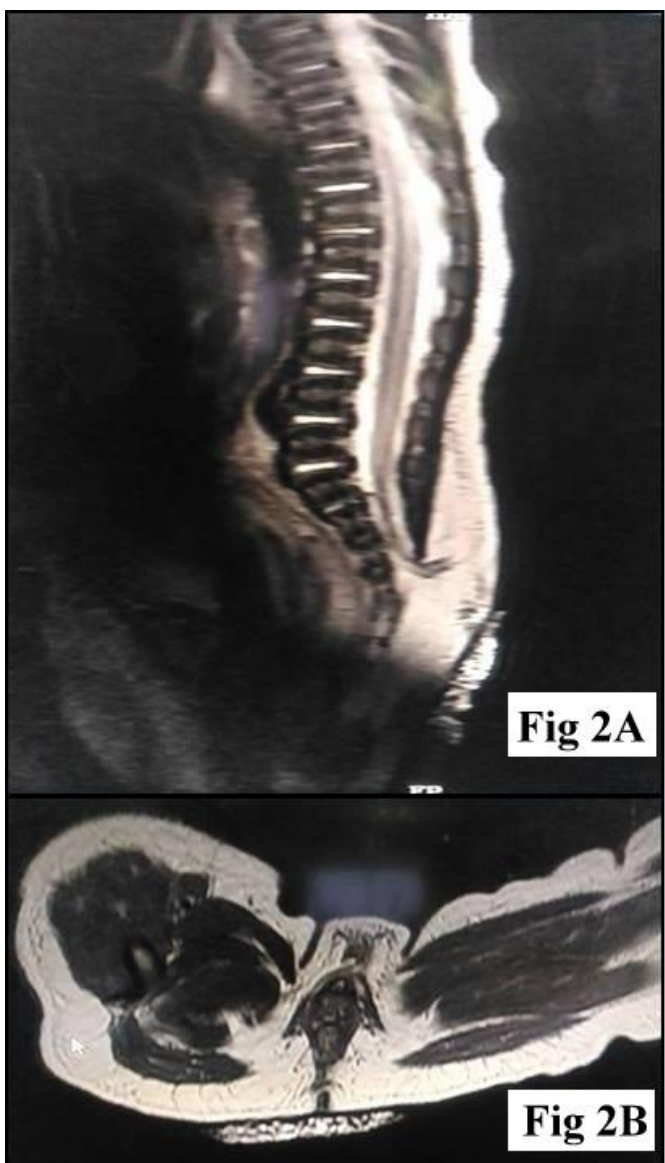

Figure 2: A (Sagital view), B (Axial view): MRI showing Spina bifida at S3, S4 and S5 levels, low lying tethered cord with conus at L5$\mathrm{S} 1$ level, well defined soft tissue lesionin right lumbosacral region on external aspect of skin wth small portion attached to skin and subcutaneous tissue at S3-S4 level.

\section{DISCUSSION}

A vestigial tail is a rare congenital anomaly and represents cutaneous marker of underlying occult spinal dysraphism.[1] During 5th and 6th week of gestation, embryo has a tail like structure which regresses by 8th week by phagocytosis.[2] Exact mechanism of formation of vestigial tail is not known. A human tail can be 'true tail' or 'pseudo tail'. A true tail is a remnant of embryonic tail [3] and not associated with any underlying malformations. [4] Caudal appendages occurring with spina bifida occulta or spinal dysraphism are pseudotails [2] as seen in our case.

Radiological investigations can differentiate between true tail and pseudo tail as the treatment of both varies. Spinal sonography can be used in early infancy [1]; MRI provides much larger field of view and is required prior to an intervention, if spinal sonogram demonstrates spinal abnormality requiring surgery.[1] True tail requires simple surgical excision [2] in contrast with pseudotail which also require surgical repair of underlying spinal abnormality which was done in our case. Long term follow up is required for neurological assessment.

It is known that maternal GDM acts as etiological factor in the pathogenesis of caudal regression syndrome. In our case a baby with vestigial tail and spina bifida was born to a mother with GDM which further suggests that GDM might be an etiological factor in the pathogenesis of vestigial tail which requires further studies to confirm.

\section{REFERENCES}

1. Cai C, Shi O, Shen C. Surgical treatment of a patient with human tail and multiple abnormalities of the spinal cord and column. Adv Orthoped. 2010;2011.

2. Sarmast AH, Showkat HI, Mir SF, Ahmad SR, Bhat AR, Kirmani AR. Human born with a tail: A case report. SAJCH. 2013; 7:38-9.

3. Pillai MK, Nair ST. A true human tail in a neonate: case report and literature review. Sultan Qaboos Univ Med J. 2017;17:109-11.

4. Turk CC, Kara NN, Bacanli A. The human tail: a simple skin appendage or cutaneous stigma of an anomaly? Turk Neurosurg. 2016;26:140-5. 